\title{
SPINOZA ET LA QUESTION ÉTHICO-SOCIALE DU DÉSIR: ÉTUDES COMPARATIVES AVEC ÉPICURE-LUCRECE ET MACHIAVEL ${ }^{\star}$
}

Laurent Bove ${ }^{\star}$

\begin{abstract}
RÉSUMÉ
"Épicure, Démocrite, Lucrèce" (dans la lettre 56 à Hugo Boxel) et "Machiavel" (dans le Traité politique) sont les deux courants de pensée que Spinoza (1966[1674], 2002 [1677]), cite de manière très élogieuse. Nous nous proposons d'étudier ici - selon une analyse comparative et à partir de la question éthicosociale du Désir - les filiations et les différences de la philosophie spinoziste avec la pensée épicurienne et la pensée de Machiavel.

Mots-clés: desir; plaisir; prudence.

\section{SPINOZA AND THE ETHICAL-SOCIAL ISSUE OF DESIRE: LUCRECIUS AND MACHIAVELLI} COMPARATIVE STUDIES OF THE PHILOSPHIES OF EPICURUS-
\end{abstract}

\begin{abstract}
Epicurus, Democritus, Lucrecius (in the letter 56 to Hugo Boxel) and Machiavelli (in the Political Treaty) are the two streams of thought that Spinoza cites in a quite eulogistic way. We propose to study here the affiliations and the differences between the philosophies of Spinoza, Epicurus and Machiavelli in a comparative analysis, departing from the ethical and social issue of desire.
\end{abstract}

Keywords: desire, pleasure, prudence.

\footnotetext{
* Cette étude a fait l'objet d'un cours donné à l'Université Fédérale Fluminense de Niteroi, les 22 et 23 août 2012, sur l'invitation de la Professeure Cristina Mair Rauter. Cette étude a pour base des textes que j'ai déjà publiés : "Épicurisme et spinozisme: 1'éthique", Archives de Philosophie, juillet-septembre 1994, tome 57, Cahier 3, p. 471-484; "Dall'innata potestas alle strategie del conatus, o l'epicureismo in moto perpetuo", in Lucrezio e la modernità. I secoli XV-XVII p. 163-178, a cura di F. Del Lucchese, V. Morfino, G. Mormino, ed. Bibliopolis, Napoli 2011; "Une ontologie politique de la durée. Spinoza lecteur de Machiavel" in Spinoza, Traité Politique, édition de L. Bove, Le Livre de Poche, 2002, Introduction "De la prudence des corps. Du physique au politique", p. 31-46.

$\star \star$ Docteur d'État (de Paris-I Sorbonne), Professeur de philosophie à l'UFR sciences humaines, sociales et philosophie - Université de Picardie Jules Verne (UPJV), chercheur à l'UMR 5037/ ENS-LSH - Ecole Normale Supérieure Lettres et Sciences Humaines.

E-mail: laurent.bove@wanadoo.fr
} 


\section{I - ÉPICURISME ET SPINOZISME}

L'épicurisme et le spinozisme se constituent dans leur lutte radicale contre un adversaire qui leur est structurellement commun: la conception téléologicodualiste et morale de l'homme et du monde et ses conséquences pratiques: l'illusion théologique et la superstition, la crainte, le manque, la tristesse, la haine.

"Aux qualités occultes, aux espèces intentionnelles et aux formes substantielles". (SPINOZA, 1966[1674], p. 300) des métaphysiques idéalistes, aux "terreurs" et aux "ténèbres de l'esprit" (LUCRÈCE, 1964, p. 54) des superstitions, Épicure, Lucrèce et Spinoza opposent la saine "étude rationnelle de la nature" (LUCRÈCE, 1964, p. 54) sans aucune adjonction étrangère, qui expulse tout arrière-monde, tout asile d'ignorance, tout mystère, au profit de la pure joie immanente du comprendre. Au manque et à la tristesse, ils opposent la plénitude du plaisir constitutif. À la haine enfin, la douce amitié. À la suite des épicuriens. Spinoza détermine donc aussi l'objet spéculatif et pratique de la philosophie comme "naturalisme" (DELEUZE, 1969, p. 361). Car de "réel" il n'y a que la nature et sa vérité, éternelles l'une et l'autre au présent.

Cela suffirait déjà pour affirmer légitimement que Spinoza et Épicure sont, par-delà les deux millénaires qui les séparent, dans un même camp et qu'il y a entre eux une véritable filiation dans le combat libérateur qui les unit contre toutes les figures de l'illusion et de l'oppression des esprits et des corps, avec pour arme, le libre, puissant et heureux exercice de la raison dans son entreprise radicale de démystification.

Épicure et Spinoza c'est la philosophie même, sa fonction vitale, sa dignité et sa grandeur, mais aussi sa force de scandale pour tous ceux qui confondent la recherche du vrai avec l'univers de leurs songes, de leurs craintes et de leurs espoirs.

Ce que nous nous proposons d'examiner ici c'est, face à un adversaire commun, la singularité de leur position respective à partir de laquelle s'éclaire, sur le terrain éthico-social - celui de la connaissance vraie sur laquelle se fonde une "conduite droite de la vie" et la recherche de ce qui nous est réellement utile - à la fois ce qui fait la filiation et la différence des philosophies.

Nous procéderons à cette confrontation des systèmes en interrogeant les deux doctrines sur leur conception du désir, du plaisir et de la béatitude qui sont, à la fois, le principe, l'objet et la fin de la pratique éthique.

Le plaisir, dit Épicure (1992, p. 221-222) dans sa Lettre à Ménécée, est:

le principe et la fin de la vie bienheureuse. Car c'est lui que nous avons reconnu comme le bien premier et connaturel, c'est en lui que nous trouvons le principe de tout choix et de tout refus, et c'est à lui que nous aboutissons en jugeant tout bien d'après l'affection comme critère. 
Le plaisir est donc principe du comportement spontané des êtres vivants, principe de choix et de refus, avant même l'intervention de la raison en tant que telle (LAERCE, 1965); la douleur, naissant de l'obstacle à la réalisation normale et normée de cette tendance naturelle: tendance ou désir par lequel s'exprime une sagesse immanente du corps vivant dont la sagesse philosophique ne sera que le prolongement rationnellement réfléchi et orienté.

La douleur dans le corps et le trouble dans l'âme sont donc les signaux d'un déséquilibre, d'un désordre auxquels la philosophie comme remède - suivant en cela les mouvements mêmes de la vie - s'efforce de répondre de manière ajustée par un retour à la norme naturelle immanente de la santé, dont les obstacles extérieurs d'une part, et les produits de l'imagination humaine, d'autre part, nous avaient éloignés.

Retour donc à l'énergie vitale naturelle dans sa libre affirmation équilibrée par le moyen de l'énergie de cette vie même comme résistance, dans son expression philosophique.

Et toute la philosophie épicurienne (comme energeia) ${ }^{2}$ se présente bien comme cette résistance vitale face à toutes les formes de déséquilibres introduites dans l'ordre naturel humain.

Disons d'ores et déjà que, sauf en philosophie où 'apprendre et jouir vont ensemble" (ÉPICURE, 1992, p. 253-255), cette dynamique du retour n'est pas pour elle-même un plaisir pur, même si elle est en elle-même suscitée par une résistance de la vie à la tristesse et à la douleur, du fait de la connaturalité du plaisir. Le mouvement de restauration présuppose le plaisir comme son principe mais il ne le manifeste pas en sa pureté dans le mouvement même (c'est la douleur qui prime!) mais seulement dans sa cessation, donc dans la satisfaction du repos et de l'équilibre retrouvés.

Lucrèce décrivant les chocs répétés et violents du dehors qu'ont souvent à subir les êtres vivants, et après avoir souligné la résistance des "mouvements vitaux qui subsistent" (LUCRECE, 1964, p. 77) et qui dès lors peuvent, dit-il, "calmer le tumulte excité, ramener chaque élément dans ses conduits naturels et domptant la mort déjà presque maîtresse du corps, rallumer ainsi la sensibilité à peu près éteinte" (LUCRECE, 1964, p. 77)... Lucrèce donc écrit qu'il n'y "a doux plaisir" que lorsque "les principes de la matière [...] reprennent leur place” (LUCRECE, 1964, p. 77).

La position épicurienne se précise d'ailleurs dans le De Natura Rerum avec l'apport explicite de la théorie du clinamen selon laquelle est posé un véritable principe de plaisir (COMTE-SPONVILLE, 1984, p. 54-56).

Lucrèce (1964) explique en effet l'activité du vivant humain non seulement par l'effet extrinsèque des simulacres qui "viennent frapper (son) esprit" (p. 140) et déterminent ainsi en lui la volonté de se mouvoir mais aussi par une "innata potestas" (p. 60), une puissance naturelle et désirante "(vim cupidam)" (p. 59) qui en elle-même est pure positivité, ne manque donc de rien et qui, dans son affirmation est apte à répondre de manière ajustée aux forces extérieures destructrices. 
Puissance donc de résistance et de combat de la Nature elle-même en chacune de ses vivantes individuations, dont l'activité n'a qu'un but: le rétablissement du plaisir en repos et de la paix. Lucrèce $(1964$, p. 60) écrit:

en dépit de la force étrangère qui souvent nous oblige à marcher malgré nous-même, nous emporte et nous précipite, il y a pourtant en nous quelque chose capable de combattre et de résister. C'est ce quelque chose, dont les ordres meuvent la masse de la matière dans notre corps, dans nos membres, la réfrènent dans son élan et la ramènent en arrière pour le repos.

Cela ne signifie cependant pas que, dans le repos, cette puissance d'affirmation et de résistance soit elle-même inactive et inutile: elle est, au contraire, l'affirmation indéfiniment renouvelée de la plénitude, activité immobile en quelque sorte, dont les dieux, "par le privilège de leur nature [...] puissants de leurs propres forces" (LUCRÈCE, 1964, p. 20), nous donnent un excellent exemple.

Le clinamen donc, chez les hommes comme chez les dieux, loin d'être le pouvoir arbitraire et obscur d'une imprévisible réponse de hasard ou du hasard, est au contraire, au cœur du vivant, cette puissance positive d'affirmation et de résistance par laquelle, selon sa déclinaison propre, chaque être s'arrache à tout destin (tant externe qu'interne) par un "mouvement qui rompt les lois de la fatalité" (LUCRÈCE, 1964, p. 59). C'est une sorte de conatus, de détermination positive selon laquelle, chaque être vivant est, de manière aussi minimale soit-elle, puissance autonome d'affirmation et de résistance dans laquelle et par laquelle, la Nature, en son affirmation, s'affecte joyeusement en quelque sorte elle-même en se déterminant positivement en chacune de ses individuations.

Le modèle d'autonomie des dieux épicuriens nous permet en ce sens de penser une première filiation théorique forte entre Spinoza et Lucrèce.

La démonstration de la proposition 4 de la partie IV de l'Éthique (SPINOZA, 1993), examine, en effet, les deux hypothèses qui pourraient, a priori, expliquer qu'un être quelconque (et Spinoza parle ici de l'homme) pourrait ne jamais périr (ou exister nécessairement toujours). Or ces deux hypothèses sont les deux conditions de la vie bienheureuse des dieux épicuriens...

- première hypothèse: soit que cet être, selon Spinoza, est capable de ne pâtir d'autres changements que ceux qui peuvent se comprendre par sa seule nature; c'est-à-dire, par conséquent, qu'il serait capable aussi de résister à toute violence et "d'éloigner de soi tous les autres changements qui pourraient naitre des causes extérieures". Spinoza fonde cette logique de l'autonomie sur sa théorie du conatus en renvoyant le lecteur aux propositions 4 et 6 d'Éthique III dans lesquelles il a posé déjà:

$-1^{\circ}$ ) que nulle chose n'a, en soi, rien qui puisse la détruire, et qu'elle ne peut donc être détruite que par une cause ou une force extérieure, 
$-2^{\circ}$ ) et que, par conséquent, autant qu'il est en elle [de puissance] elle s'efforce indéfiniment de persévérer en son être en résistant activement à tout ce qui contrarie son affirmation.

- deuxième hypothèse de la démonstration de la proposition 4 d'Éthique IV. Pour qu'un être ne périsse jamais il faudrait que l'ordre de la nature soit disposé d'une manière telle que cet ordre soit indéfiniment favorable à la conservation d'un seul être - en l'occurrence l'homme -. Or, en tant que mode fini de la Nature, l'homme ne peut pas ne pas pâtir d'autres changements que ceux qui peuvent se comprendre par sa seule nature...

Cette dernière proposition rend alors les deux hypothèses (qui feraient effectivement de l'homme un être immortel ) absurdes. Mais cette absurdité ne porte cependant pas sur la logique intrinsèque de la persévérance (abstraction faite des causes extérieures) qui, elle, est bien une logique de l'autonomie c'est-à-dire de la conservation indéfinie de la chose dans et par ses effets.

Et c'est cette logique de l'autonomie (ou de la "cause adéquate", Éthique III, définition 1, qui est aussi la dynamique d'une ontologie de la durée, comme continuation indéfinie d'une existence quelconque, suivant la définition 5 de la partie II), cette logique est la logique même, indéfiniment continuée, des dieux épicuriens telle que Lucrèce nous la rapporte dans le De rerum natura. [réf.]

Lucrèce (1964) insiste bien d'abord en effet, sur l'autonomie de la persévérance des dieux dont la nature propre est, dit-il, ipsa suis pollens opibus (I, 44-49 et II, 646-651), c'est-à-dire forte de ses propres ressources. Mais Lucrèce (1964) ajoute que les dieux, bénéficient aussi, de plus (et ce sera la seconde hypothèse envisagée par Spinoza), d'une nature entièrement disposée en faveur de leur persévérance dans "un ciel toujours pur [qui] les couvre et leur sourit de sa fluide clarté. Tout est sans cesse offert aux dieux par la nature, poursuit-il, [et] aucun trouble jamais n'effleure la paix de leur ame" (III, 21-24). Dans ce même livre, Lucrèce envisageait déjà lui-même les conditions d'une durée éternelle d'un corps quelconque qui serait capable de repousser indéfiniment les coups venant de l'extérieur et capable ainsi de ne rien laisser pénétrer en lui qui puisse détruire l'union étroite de ses parties. Or, de ce point de vue, Lucrèce concluait déjà que l'âme humaine n'était pas, par elle-même (par ses propres forces) ni, non plus, de par ses conditions effectives d'existence, "à l'abri des choses de la vie" et que, par conséquent, elle ne possédait pas les conditions nécessaires de l'immortalité (III, 806-829 et V, 351-379)...

Le texte de Spinoza de la démonstration de la proposition 4 d'Éthique IV, fait donc parfaitement écho aux développements de Lucrèce, en suivant une même logique et en aboutissant à une même conclusion.

Un autre texte de Spinoza fait aussi écho au modèle d'autonomie des dieux épicuriens. C'est l'hypothèse d'un Adam qui aurait été réellement cet être parfait d'intégrité que les théologiens imaginent. Dans l'article 6 du chapitre II du Traité politique, Spinoza (2002) dit, en effet, que si Adam avait pu exercer la science et la prudence (sciens et prudens) qu'on lui suppose, il aurait été alors impossible de 
le faire dévier, en quoi que ce soit de sa libre et éternelle nécessité. Car chez Adam, comme chez les dieux épicuriens, il y aurait eu identité parfaite de la puissance, de l'existence et de la liberté (si l'on ne confond pas la liberté d'Adam avec la contingence du libre arbitre mais que l'on entend au contraire par liberté, une puissance, une vertu, une perfection). Dans l'article 9 du chapitre II du Traité politique, le lien nécessaire entre l'effectivité de cette liberté et la capacité de résister, sera attribué à l'homme lui-même qui, ne "relève de son propre droit" (c'est-à-dire n'est sui juris) que "dans la mesure où il peut repousser toute violence [vim omnem repellere]", c'est-à-dire dans la mesure où il peut activement et puissamment résister à tout ce qui est contraire à son effort de persévérance in suo esse (ce que théoriquement aurait pu et aurait dû faire le premier homme, et ce que pourraient faire aussi les dieux épicuriens, bien que cette situation défavorable ne se présente pas à eux puisque la nature extérieure leur est toujours clémente).

Si l'on articule alors la théorie du clinamen avec la pure positivité de l'innata potestas, comme puissance naturelle résistante et désirante, on comprend que le clinamen épicurien ait pu être lu par Spinoza comme un équivalent de ce que lui-même entendait par la positivité intrinsèque d'une détermination continuée. J'ai déjà développé cette hypothèse dans le chapitre VI de La stratégie du conatus en commentant les lettres 54 et 56 à Hugo Boxel (BOVE, 2012[1996], p. 147 et seq.), je n'y reviens donc pas ici.

Il y a aussi chez Spinoza - en un autre sens il est vrai car là il ne s'agira pas seulement d'un "retour" - un véritable principe de plaisir, inhérent au conatus, qui explique le comportement des êtres vivants à partir d'un certain degré de complexité de leur corps, qui les rend capables, à la fois, d'éprouver joie ou tristesse et de garder ces affects en mémoire.

Arrêtons-nous sur la question de la mémoire dans son rapport au plaisir car nous allons la retrouver dans un rôle essentiel au sein de l'éthique épicurienne.

La constitution d'une mémoire s'explique dynamiquement en effet, chez Spinoza, par l'effort que nous faisons pour conserver seulement ce qui augmente notre puissance d'agir, et s'accompagne ainsi d'un affect de joie. C'est-à-dire que nous nous efforçons toujours de conserver ce qui nous est apparu utile et convenant à notre nature, avec le plaisir comme critère.

Et, "agir par vertu absolument" ne consistera en rien d'autre que d'"agir vivre et conserver son être [...] sous la conduite de la Raison, d'après le principe de la recherche de l'utile propre"-comme le dit la prop. 24 d'Eth IV ou, précise la démonstration, d'agir "par les lois de sa nature propre" c'est-à-dire de manière autonome.

Dans la constitution d'une éthique de l'autonomie, nous passons donc nécessairement de la recherche de ce qui est apparu utile, sous la détermination de notre mémoire, à la recherche de ce que nous connaissons, en vérité, nous être réellement utile sous la détermination de notre Raison.

Mais c'est, en premier lieu, vers "ce qui accroît ou seconde la puissance d'agir du Corps" que nous nous efforçons spontanément (Eth.III, 12). 
Et nous nous efforçons naturellement aussi quand, sous 1'effet de causes extérieures qui nous sont contraires, notre âme "imagine ce qui diminue ou réduit la puissance d'agir [de notre] Corps [...], de [nous] souvenir de choses qui excluent l'existence de ce qu'elle imagine" (Eth. III, 13 et aussi sc. ).

Selon cette logique, le conatus "résiste alors comme un fleuve exerçant une pression sur un barrage et qu'il tend à éliminer en suscitant ce qui l'exclut", suivant l'image dynamique d'une résistance-active auto-organisatrice proposée par Alexandre Matheron (1986a, p. 211) en illustration de cette prop. 13.

C'est donc à un réinvestissement de l'image de l'objet qui a procuré la satisfaction que nous nous efforçons naturellement et cet effort, sous la détermination du plaisir, définit la Mémoire. Corrélativement nous résistons aussi à l'oubli inéluctable, sous la pression des images du présent qui excluent les images du passé, de l'image accompagnée de joie. Cet effort est résistance à l'extinction d'un affect joyeux, c'est-à-dire résistance à la tristesse et à ses causes, et tendance à réactiver l'intensité de l'image favorable contre une réalité ou d'autres images - qui nous sont à présent nuisibles. Il s'agit donc d'oublier, de refouler, "d'écarter et de détruire" la représentation nuisible au profit d'une autre, investie d'un affect joyeux (Eth. III, 28).

C'est dans cette stratégie spontanée de résistance-active du conatus, qui n'est pas simplement de préservation d'un "état" mais tendance affirmative d'auto-organisation de la vie selon la dynamique même de l'essence de l'homme ("de la nature de laquelle suit nécessairement ce qui sert à sa conservation", Eth. III, $9 s c$.), que la Mémoire prend, chez Spinoza, valeur à la fois de matrice (condition de possibilité), de moyen, mais aussi d'arme, pour la joie contre la tristesse.

La joie comme la tristesse sont donc les premières données stratégiques qui orientent le dynamisme, en lui-même sans fin, du conatus.

Nous sommes là, semble-t-il, très proche d'Épicure qui a montré combien la mémoire peut être un instrument essentiel de notre bonheur,

- premièrement, en maintenant toujours présentes les doctrines "générales et principales" nécessaires à la vie heureuse. C'est une condition nécessaire de l'ataraxie comme l'indiquent les lettres à Hérodote, à Pytoclès et à Ménécée.

Et l'on pense parallèlement aux conseils pratiques de mnémotechnie, à ces "principes assurés de conduite" qu'il faut "imprimer en notre mémoire", que donne Spinoza dans le sc. de la prop. 10 d'Eth. V, qui correspondent assez bien à cette mémorisation souhaitée par Épicure et qui doit constituer un véritable habitus qui assure à la fois la rapidité du jugement et de la décision pratique et par conséquent l'ajustement éthique en toutes situations. Ici la sagesse pratique (la phronésis épicurienne) a pénétré par l'habitude et ses contractions la spontanéité apparente de la vie. 
On pense aussi aux sept dogmes de foi enseignés par le Christ, qui "sauvent" s'ils sont crus vrais et appliqués dans le plein consentement de l'âme, et que Spinoza (2009[1670]) présente dans le Traité théologico-politique, comme le véritable septuple remède à la condition humaine ordinaire.

Mais dans ce dernier cas, le remède - qui vaut essentiellement par son efficacité thérapeutique et non par sa vérité - s'applique seulement aux ignorants, c'est-à-dire à des hommes dominés par l'imagination... et dans le cas précédent celui du sc. D'Eth. V, 10 - la technique est conseillée "tant que nous n'avons pas une connaissance parfaite de nos affects".

Ce qui signifie que pour Spinoza c'est essentiellement la connaissance adéquate de nos propres affects, comprise dans une même dynamique de l'autonomie dans notre pensée comme dans notre corps, qui est éthiquement/réellement transformatrice, salvatrice... et non la mémoire d'une doctrine philosophique même vraie!

Mais il est toujours possible, il est vrai, que la joie passive et l'imagination soient une voie dynamique de passage à la joie active accompagnée d'idées adéquates - nous y reviendrons...

- deuxièmement la Mémoire, comme l'oubli, peuvent être aussi, pour Épicure, un instrument volontaire et essentiel de notre bonheur en ensevelissant "nos malheurs dans un oubli perpétuel", 7 ou en maintenant présents les plaisirs passés afin de contrebalancer les douleurs actuelles, comme l'écrit Épicure à Idoménée. ${ }^{8}$ Vers le passé, le sage ne se tourne qu'utilement et avec gratitude (charis).

Les prop. III, 9 et $s c$., II et $s c$., 12, 13 et $s c$. dans lesquelles peuvent se lire, avec la théorie du conatus, les éléments d'une stratégie de résistance active de la vie, inhérente à la genèse et à l'activité de la Mémoire et de l'oubli, pourraient servir de fondement ontologique dynamique à la fonction éthique de la Mémoire dans la doctrine épicurienne.

Ce qui sépare cependant Spinoza d’Épicure sur la question du plaisir - et du désir - c'est que si le plaisir est pour Spinoza connaturel à l'être humain, il n'est cependant pas contradictoire, même dans la Béatitude, avec un dynamisme essentiel et productif qui, loin seulement à la fois de répéter l'identité à soi de l'ataraxie, et de la défendre, produit effectivement dans le rapport à autrui, l'humanité de l'homme dans une histoire à la fois collective et interminable.

Chez les épicuriens, l'innata potestas est une puissance d'affirmation et de résistance, certes active, en ce qu'elle est constitutive, comme l'a montré V. Brochard, mais d'une énergie essentiellement en repos (Cf. BROCHARD, 1912, p. 266-267).

Le conatus spinoziste est lui, au contraire, essentiellement dynamique même si c'est dans son activité intrinsèque-autonome que s'expérimente aussi - vis-à-vis des forces extérieures - une forme de stabilité et de repos dont nous jouissons dans la Béatitude. 
C'est la différence d'une persévérance in suo statu (en son état) caractéristique de la katastéma épicurienne, et d'une persévérance "en son être" (in suo esse) caractéristique du spinozisme. Car 1"être" est ici le mouvement réel du réel lui-même en son auto-constitution, au principe du procès éthique, dans l'autoorganisation relationnelle et autonome du mode humain (sur la base des lois des affects, imitation, pitié, ambition de gloire et du principe de plaisir).

C'est la différence, dans l'entreprise éthique, de deux types de constitutions et de deux types de stratégies:

D'abord, une stratégie épicurienne du "retour" ou de la "restauration" selon une norme de nature implicite toujours-déjà-là, elle-même constitutive. Logique de la purgation, de la délivrance, de la pureté du plaisir reconquise et/ ou de la reterritorialisation sur un socle primitif individuel dont le modèle est l'atome. Même si cet "individualisme" est fortement contesté, mais a posteriori, par l'importance accordée à l'amitié qui s'avère alors aussi nécessaire voire aussi éternelle que la sagesse elle-même, nous rapprochant ainsi de l'amour spinoziste du prochain.

La Sentence Vaticane 52 met en effet en relation l'amitié et un "réveil" de notre nature (en son essence, semblable à celle des dieux), qui nous permettrait, comme les dieux, de ne voir dans nos semblables essentiellement que des amis alors que l'existence concrète nous les présente, au contraire, comme nos pires adversaires (cf. Conche, note, p. 240). L'amitié, comme la sagesse, serait ainsi la voie vers l'essentiel retrouvé. Dans l'amitié épicurienne c'est donc la vie qui s'aime elle-même, se désire elle-même éternellement. En ce sens l'amitié comme la sagesse est un bien immortel en ce qu'elle enveloppe une vérité éternelle, la norme même de la santé du corps collectif, son équilibre naturel. La doctrine épicurienne de l'amitié ferait donc signe, comme chez Spinoza, vers un amour de soi qui enveloppe l'amour d'autrui dans un commun amour de la vie en nous, par nous et à travers nous.

L'amitié épicurienne exprime, de plus, une double résistance vitale au pouvoir et aux déséquilibres que celui-ci introduit dans la vie des hommes: au pouvoir de la Fortune tout d'abord (et le Sage, qui se dresse en face de la Tychè comme un combattant - suivant l'expression rapportée par D.L. X, 120 - trouve en ses amis de solides alliés); mais aussi aux pouvoirs instaurés par les hommes eux-mêmes qui dissolvent et pervertissent la vie. L'appel à la sociabilité est alors, chez les épicuriens comme chez Spinoza, un appel à la résistance aux pouvoirs: chez les épicuriens, par fuite ou retrait, afin de sauver le naturel-essentiel, pour vivre, ailleurs que dans l'espace politique, la douceur de la collectivité selon une sociabilité retrouvée, restaurée; chez Spinoza, au cœur même du politique, par la constitution collective d'une socialité dont la puissance, comme l'a dit Toni Negri, s'affirme contre les pouvoirs.

- ensuite, une stratégie du conatus spinoziste, constituante de sa propre norme dans une dynamique relationnelle d'affirmation absolue de l'existence de la modalité singulière. Procès causal de la cupiditas, "sans principe ni fin" ni modèle, d'adéquation ouverte de l'essence et de l'existence, en progrès permanent.

Fractal, Rev. Psicol., v. 24 - n. 3, p. 443-472, Set./Dez. 2012 
Stratégie constituante donc de l'infinitude actuelle (Éth. I. 8 sc.) en sa détermination positive causale singulière (Spinoza) contre stratégie téléologicothérapeutique ${ }^{9}$ de l'être-fini-individuel, dans la reconnaissance salutaire de ses limites, et orientée par un modèle "naturel" de la santé (Épicure). L'éloge que Lucrèce fait de la prudence est essentiellement en effet celle d'une "prudence en acte dans la connaissance des limites", limites naturelles de l'être humain.

Dynamique de la simplification de la vie, du retour à l'élémentaire, à l'essence individuelle du désir et du plaisir contre dynamique de la complexification des Corps et le perfectionnement indéfini des esprits (tant individuels que collectifs) (Eth. IV, 38 et dém. et II 13 sc., 14 ; IV, 45 sc. du coroll. 2 ; IV, app. ch. 27). Le conatus spinoziste produit de l'essentiel, il ne restaure pas, il construit. ${ }^{10}$

C'est dire que Spinoza, contrairement à Épicure, n'a pas découvert et révélé au monde les bornes (finem) légitimes et naturelles du désir, comme Lucrèce en fait l'éloge à l'auteur de la Lettre à Ménécée. ${ }^{11}$ Si pour Épicure comprendre une chose c'est en reconnaître ses limites, pour Spinoza c'est au contraire la connaître adéquatement selon l'infinitude ou l'éternité qui la constitue dans son dynamisme causal essentiel. ${ }^{12}$ Chez Épicure - et plus nettement encore chez Lucrèce - c'est la crainte de la mort qui engendre l'illimitation des désirs. Chez Spinoza c'est l'infinitude actuelle, l'affirmation absolue de l'existence en une individuation singulière... Même si l'illimitation des désirs vains doit aussi s'expliquer, comme chez Épicure, par nos opinions fausses.

Mais ce n'est pas l'opinion fausse qui fait la réalité de l'illimitation, bien au contraire. La connaissance vraie ouvre "adéquatement" à l'illimitation des désirs et des plaisirs qui s'accorde ainsi avec la mesure, la tempérance des désirs sans excès dans le mouvement réel du réel en son auto-organisation éthique de la singularité.

La notion d'illimitation doit être cependant précisée. Elle enveloppe pour les épicuriens le mauvais infini, celui à la fois du fantasme qui fait du désir un vase qui ne peut jamais être rempli... ${ }^{13}$ et par conséquent, de la multiplication à l'infini des désirs et des plaisirs. la recherche illusoire d'un contentement impossible. Pour Spinoza, si les désirs sont réellement illimités, c'est,

1) du fait même de la présence actuelle et intensive de l'infini dans le fini qui fait en tout désir, de la jouissance de soi qu'accompagne nécessairement l'idée adéquate de ses propres affects, une expérience réelle de l'infinitude et de l'éternité,

2) du fait aussi des parties du Corps humain qui certes ne sont pas infinies mais enveloppent un très grand nombre d'aptitudes qui peuvent toujours être augmentées, et on ne sait pas ce que peut un Corps !

Et cette augmentation n'introduit pas nécessairement le mauvais infini. C'est sans excès que les plaisirs et les désirs du sage sont à la fois infinis de nature et sans limites dans leur diversité.

La Nature, en son affirmation absolue et toujours singulière de l'existence et/ou des existences (conatus ou clinamen), c'est ce qui résiste en nous à l'illusion et à la tristesse, aux faux infinis des mythes et des désirs vains. 
Épicure et Spinoza sont là-dessus d'accord. Mais pour Spinoza la Nature n'est pas ce qui résiste a l'illimitation, tant interne qu'externe, de nos actions et de nos désirs, mais au contraire ce qui la produit et qui, dans cette surabondance même, qui absolument parlant est "sans excès", nous permet de résister à cette autre illimitation, elle, asservissante qui est celle des désirs vains....

II y a bien chez Spinoza une distinction du plaisir - ou de la joie - en mouvement (qui accompagne l'augmentation de notre puissance d'agir) et d'un plaisir en quelque sorte en repos (catastématique dirait Épicure) dans la possession formelle de cette même puissance dans le troisième genre de connaissance (Eth. V, 33 sc.). Mais même dans la plénitude de sa perfection en repos, aucune limite n'est pourtant fixée au dynamisme productif du désir, et au plaisir, qui, en dehors de tout excès mais aussi de toute borne, se déploient à présent - et au présent - dans une extension toujours plus grande des aptitudes à être affecté et à affecter de notre corps et de la perfection de notre esprit, suivant la voie indiquée par le scolie de la prop. 39 d'Eth. V. C'est, dans le procès éthique, la dynamique accumulative sans fin, en boucle récursive, de la vertu, déjà définie par Eth. IV, 20. Éthique de la quantité en dehors de tout manque (Eth. IV, 38) fondée au contraire sur la prodigalité infinie de la Nature en son affirmation déductive et/ou en l'infinité infinie de ses individuations,

Le désir se déploie ainsi en chaque être selon une puissance pratique stratégique d'affirmation et de résistance-constitutive, sans modèle ni limite (Eth. III, 2 sc. et V, 39 sc.). Comme le dit Alexandre Matheron (1986b, p. 179), "Spinoza en toute rigueur et théoriquement ne sait pas ce que c'est que l'homme et il s'en passe très bien"... et cette ignorance fondée est libératrice. Elle ouvre l'éthique et la politique à l'histoire c'est-à-dire à la pratique collective et constituante de la Nature elle-même;

- D'une part, tous les désirs ou affects que nous avons et que nous aurons peuvent s'exercer pleinement et sans excès, activement donc, selon la dynamique même de la Raison (Eth. IV, 59 et dém. 2, V, 4 sc.),

- D'autre part, "plus nous connaissons les choses singulières, plus nous connaissons Dieu" (Eth. V, 24) et par là même plus nous désirons connaître les choses par le troisième genre de connaissance $(E t h$. V, 26)... et cette dynamique de la connaissance et de l'amour par laquelle et dans laquelle nous jouissons de l'infini enveloppe en elle un temps indéfini et constituant.

Bien que pleinement satisfait, le sage ne persévère donc pas seulement "en son état" de repos, à l'abri des limites déjà tracées par la nature. Il ne cesse pas, au contraire, ouvert à l'infinie diversité de la nature - non-humaine et humaine - de progresser adéquatement, d'étendre sa vie, sa perfection, ses désirs et ses plaisirs avec de nouvelles idées vraies. ${ }^{14}$ Non seulement la plénitude se nourrit de l'infinie diversité du réel, des choses comme des hommes, non seulement elle résiste à ce qui pourrait, de l'extérieur, l'ébranler, mais elle est aussi féconde, dans sa résistance même, productrice de nouveautés dans sa reproduction. Ce n'est donc pas la seule satisfaction normée des désirs qui conduit à la plénitude, 
mais c'est la fécondité de la plénitude elle-même qui multiplie indéfiniment les désirs, en nous et par nous, nécessairement devenus nécessaires au prolongement et à l'expansion sans bornes de notre perfection humaine.

Nous nous sommes jusqu'à présent situés dans la dynamique de la Béatitude elle-même. Mais c'est aussi par la dynamique de la simple joie, même passive, que s'explique chez Spinoza l'accès à la sagesse. C'est le passage de 1' Hilaritas (affect certes passif mais par lui-même "bom", "sans excès" et qui s'accorde ainsi avec la raison; Eth. IV, 42 et 59 dém.), à la joie active du second et du troisième genre de connaissance, c'est-à-dire effectivement accompagnée d'idées adéquates. II y a là chez Spinoza une véritable dynamique plurielle de la joie ou de la douceur même de vivre qui accompagne et suscite toutes nos activités, quelles qu'elles soient, qui, dès qu'elles s'effectuent, affirment et expriment "absolument" notre plaisir d'être et d'être-avec. C'est le procès le plus direct de composition et d'organisation de la modalité vertueuse comme subjectivité éthique relationnelle qui est aussi celui de la raison. La logique de l'Allégresse est une dynamique de la perfection infinie: c'est l'épicurisme multiplié et mis en mouvement sans fin. ${ }^{15}$

Spinoza ne distingue pas en effet entre des désirs "naturels et nécessaires" et des désirs "vains" 16 mais entre des affects passifs tristes et des affects passifs joyeux, puis au sein de ces derniers des affects qui expriment un déséquilibre du Corps du fait d'un plaisir excessif ne concernant qu'une de ses parties - c'est la Titillatio (Eth. III, II sc.; IV, 43 et dém.); ou des affects qui expriment au contraire un équilibre global du Corps du fait de causes extérieures totalement favorables - c'est l' Hilaritas (Eth. III, II sc. ; IV, 42 et 44 sc.). Et ce dernier type d'affect passif-joyeux-équilibré permet un passage direct aux affects actifs, nécessairement joyeux et équilibrés par leurs propres forces - c'est la Beatitudo (Eth. IV, 45 sc. du coroll 2 et 59 dém.).

L'Allégresse ou Hilaritas est, en effet, dans la stratégie du conatus spinoziste, l'effet d'une dynamique d'affirmation doublement équilibrée:

- équilibrée de manière interne tout d'abord puisque cet affect

consiste en ce que toutes (les) parties (du Corps) sont pareillement affectées, c'est-à-dire (prop. II partie III) que la puissance d'agir du corps est accrue ou secondée de telle sorte que toutes ses parties conservent entre elles le même rapport de mouvement et de repos (Eth. IV, 42 dém.),

- équilibrée ensuite avec l'extériorité et plus particulièrement avec les autres hommes avec lesquels le Corps établit des rapports d'échanges harmonieux... corrélatifs en notre âme de la production des notions communes.

Et cette situation globale d'équilibre exprime une joie qui, par sa seule dynamique affirmative, conduit l'homme à la Béatitude, comme on peut le lire dans le scolie du coroll. 2 de la prop. 45 et la dém. de la prop. 59 d'Eth. IV. 
Ainsi, si chez Épicure la Béatitude est un état plein et équilibré, sur le modèle corporel du rassasiement du ventre - qui ne saurait donc être par là même augmenté mais seulement préservé, maintenu - et chez Spinoza, au contraire, un mouvement, celui aussi plein et équilibré du réel lui-même, producteur en nous et par nous de nouveaux désirs et de nouveaux plaisirs, dans les deux cas il s'agit de l'avènement d'une stratégie parfaite de la vie en son auto-organisation autonome et relationnelle dont les dieux sont, pour l'épicurien, le parfait modèle. Stratégie parfaite qui, dans l'activité immobile du repos (Épicure) ou l'immobilité active et féconde de la sagesse (Spinoza) met fin à l'agitation d'une vie de crainte et d'ignorance "ballottée par les causes extérieures", suivant la métaphore épicurienne de la tempête employée deux fois dans l'Éthique, dans le scolie de la prop. 59 partie III et le dernier scolie de la partie V qui en est aussi la conclusion.

On peut donc voir légitimement dans le spinozisme une sorte d'épicurisme, mais un épicurisme de la puissance de la complexité que Spinoza a ouvert au mouvement sans fin de l'anthropogenèse et de l'histoire.

Dans l'accès à la vérité du Réel, son repos et/ou son mouvement essentiel (ataraxie et acquiescentia in se ipso, ou encore Contentement "objet suprême de notre esperance", Eth. IV, $52 s c$., dans l'adéquation à soi et à la vie), ce à quoi nous convient Épicure et Spinoza, c'est à une expérience singulière de l'éternité de la Nature en nous et par nous. Nous craignions la mort, nous souhaitions être immortels : la Béatitude nous délivre définitivement de cette crainte et de cet espoir.

S'il faut certes, sur le conseil d'Épicure - et tant que nous n'avons pas encore, dirait Spinoza, une connaissance suffisante de nos propres affects - méditer sur la mort pour en comprendre à la fois le néant et l'innocence et délivrer ainsi progressivement la vie humaine du fantasme de l'immortalité, la vie libérée affirme autre chose que cette absence du désir d'être immortel et de la crainte de l'au-delà. Elle affirme pleinement son autonomie c'est-à-dire son infinitude et son éternité actuelles. ${ }^{17}$ Car c'est à présent, et dans la durée du présent, l'expérience réelle de notre éternité qui nous délivre activement du désir d'être immortel, et non plus des "raisonnements et des discours" qui n'étaient que les moyens à l'aide desquels, comme Épicure selon Métrodore, nous pouvions “monter vers l'infini et l'éternité".

Spinoza et Épicure se rencontrent donc au faite d'une sagesse naturaliste de l'immanence qui est, au présent, l'expérience de l'éternité du réel dans sa connaissance adéquate selon la dynamique autonome d'une individuation singulière par laquelle, dès ici et maintenant, au cœur de nous-même, la mort est vaincue. Car "il ne ressemble en rien à un vivant mortel, l'homme vivant dans des biens immortels", comme le dit la Lettre à Ménécée. ${ }^{19}$

Ainsi, pour autant que nous suivons leur exemple et leur enseignement, Épicure comme Spinoza nous font donc entrer, chacun à sa manière mais tous deux sans sortir de ce monde qui est le seul monde, "en possession de l'éternité", et par là même "nous tirent d'entre les morts" suivant les fortes expressions de la lettre 75 à Oldenburg à propos de la puissance salvatrice de l'enseignement du Christ, ce philosophe spinoziste par excellence, si l'on en croit Spinoza lui-même... 
Certes, pour que l'épicurien rejoigne le spinoziste, il faudrait libérer en lui le mouvement réel du réel en sa multiplicité productive, effacer la dernière figure du vide ou les modèles d'ordre et d'individualisme dans lesquels s'enferme encore sa sagesse. Mais il est vrai aussi que l'épicurisme aujourd'hui, comme aux temps d'Épicure et de Lucrèce, demeure toujours à la fois une tentation et une alternative matérialiste possible de retrait, dans la sagesse et l'amitié, contre la misère de l'histoire. Alors que le spinozisme est beaucoup plus ambitieux puisqu'il prétend ouvrir, pour tous, une alternative historique éthico-politique contre l'histoire de la misère.

Entre épicurisme et spinozisme, il y a donc sûrement affinité spirituelle, homologie certaine entre projet, thèmes et systèmes de relations mais, d'une part les problématiques des deux philosophies sont fort différentes, d'autre part, et de ce fait même, les termes d'un auteur à l'autre changent de sens; même s'ils ne sont jamais réellement tout à fait contradictoires et s'ils gardent aussi toujours une certaine familiarité ou même parfois une possibilité d'articulation voire d'intégration d'un système à l'autre.

"Il faut rire et ensemble philosopher" écrivait Épicure. ${ }^{20}$ Et sur ce point, comme sur beaucoup d'autres, Spinoza de l'approuver entièrement... car "le rire, dit l'auteur de l'Éthique, est une pure joie" et nous savons combien "plus grande est la joie dont nous sommes affectés, plus grande la perfection à laquelle nous passons, plus grande (enfin est) notre aptitude à participer de la nature divine" (Eth. IV, 45 sc. du coroll 2 et app. ch. 31 )... ou, suivant la belle conclusion de la Lettre à Ménécée, à "vivre comme un dieu parmi les hommes". ${ }^{21}$ En s'efforçant, ajoute le spinoziste, autant que faire se peut, de hisser tous les hommes au rang des dieux.

\section{II - Une ontologie POlitique de la durée: Spinoza lecteur de MaChiavel}

\section{1) Individu et conservation: la perspective polémologique}

C'est sur cette base de la conception que Spinoza nous donne de l'individu - en tant que corps - et de sa conservation, que nous pouvons juger combien sa lecture de Machiavel (déjà sensible dans le Traité Théologico-politique et évidente dans le Traité Politique où le Florentin est explicitement cite) ${ }^{22}$ a dû jouer un rôle essentiel dans l'approfondissement de son approche conceptuelle de l'individu et de sa persévérance: "Ce qui constitue la forme d'un Individu, écrit Spinoza, consiste en une union de corps; or en dépit d'un continuel changement de corps, cette forme (par hypothèse) est retenue" (Éth. II, dém. lemme 4).

La loi de conservation de la forme est donc l'essence même de l'individu, en tant qu'il s'efforce de persévérer en son être, qui "exprime", "en un mode certain et determine", la nature, l'essence ou encore la puissance de Dieu "cause de toutes choses" (Éth. I, 36 dém). Cette loi, c'est celle du conatus lui-même. La lecture de Machiavel va confirmer pour Spinoza l'identification de l'essence actuelle (le conatus) et d'une logique de l'existant s'efforçant de durer, qui est celle d'une dy- 
namique stratégique déterminée d'affirmation et de résistance. C'est ce que nous pouvons examiner à partir de trois concepts clés que l'on trouve aussi bien chez Machiavel que chez Spinoza: les concepts de nécessité, de prudence et de vertu. ${ }^{2}$

Envisageons tout d'abord, de manière générale, le regard que Spinoza porte sur Machiavel. Avec les matérialistes antiques, ${ }^{24}$ Machiavel est le penseur à qui Spinoza réserve le plus bel éloge de son œuvre, en deux articles du Traité Politique: 1'article 7 du chapitre V et l'article 1 du chapitre X. Comme c'est aussi le cas vis-à-vis des matérialistes antiques, Spinoza reconnaît tout d'abord en Machiavel un allié dans la lutte radicale qu'il mène contre un adversaire qui leur est structurellement commun, le théologien. Et au-delà du personnage-théologien (juif ou chrétien, catholique ou réformé) une structure idéologico-pratique: la conception téléologique et morale de l'homme et du monde.

Certes, Spinoza, lorsqu'il parle de l'œuvre de Machiavel, réserve son jugement (et nous reviendrons sur les raisons de cette réserve) mais c'est pour aussitôt ajouter qu'il tient quand même Machiavel pour "un homme sage" (viro sapiente) c'est-à-dire savant, intelligent et plein de bon sens. Il soulignera aussi la sagacité et la prudence de ses conseils (viro prudentissimo), et de sa très grande pénétration d'esprit (acutissimus Machiavellus) dans l'examen, à la fois,

$1^{\circ}$ ) des moyens dont doit user un prince "mû par le seul désir de domination" (TP V, 7) pour constituer sa souveraineté et la conserver,

$2^{\circ}$ ) 1'examen aussi des moyens dont un peuple, mû par le seul désir de ne pas être "commandé et opprimé des plus Gros" (suivant l'expression du chapitre IX du Prince (MACHIAVEL, 1952a, 1952b), ${ }^{25}$ doit également user pour consolider et préserver sa liberté.

Spinoza, lecteur de Machiavel, pointe donc ces deux logiques a priori antagonistes (puisque par le seul désir de domination "il s'agit d'avoir des esclaves [servos potius] plutôt que des sujets [quam subditos habere]"), comme l'objet propre de l'analyse politique machiavélienne... dont il suppose - en même temps - qu'elle s'exerce au service du camp de la liberté : "Je penche plutôt vers cette interprétation de l'œuvre de cet homme si sage" (en TP V, 7).

Remarquons que ces deux logiques contradictoires sont deux logiques du désir (ou de deux "humeurs différentes") ${ }^{26}$ et, du point de vue de Spinoza, deux expressions d'une seule et même nécessité dynamique qui est celle des conatus.

Nous savons que sans la puissance de résistance, le droit de nature individuel, que Spinoza identifie au conatus lui-même (en TP III, 18), ne serait que théorique, plus “opinion" que réalité, "puisqu' on n'a aucune façon sûre de le faire prévaloir" (en TP II, 15). Chacun, en effet, relève effectivement "de son propre droit, aussi longtemps seulement qu'il peut se garder de l'oppression d'autrui" (en TP III, 18) et, "résister à toute violence". Chacun se donne ainsi d'autant plus de droit que, comme l'écrivait Machiavel, il se "donne des moyens de s'agrandir, et de prendre des forces pour repousser quiconque voudrait attaquer, et pour anéantir qui voudrait s'opposer à [son] accroissement de puissance". ${ }^{27}$ 
C'est, de fait, la dynamique même d'affirmation et de résistance du conatus que Spinoza pouvait lire chez Machiavel. ${ }^{28}$ C'est toujours aussi sous la détermination de la nécessité, celle des conditions extérieures et/ou celle de leurs propres désirs, que les hommes agissent, écrit Machiavel. ${ }^{29}$ Spinoza ne dit pas autre chose en identifiant le conatus, ou la nécessité de la nature humaine, avec la nécessité même des passions (TP I, 5). Particulièrement l'ambition (TP VII, 6-10) qui fait que les hommes "sont par nature ennemis [et] qu'ils conservent cette nature, même lorsqu'ils sont unis par des lois" (TP VIII, 12). Pour Machiavel comme pour Spinoza, la nécessité du réel c'est donc, à la fois, celle de la dynamique d'individuation de l'affirmation/conservation (des conatus), et celle des rapports de forces que ces affirmations plurielles de puissances engendrent - si l'on peut dire - nécessairement.

C'est dans cette perspective polémologique, avec son double aspect agonique et agonistique, que nous plonge l'Éthique dès ses premières lignes. C'est en effet dès la seconde définition que Spinoza souligne combien chacune de nos idées comme chacun de nos actes rencontre nécessairement, en dehors de lui, d'autres individus de même nature - c'est-à-dire d'autres idées et d'autres corps qui les limitent et qui leur font obstacle. Après la définition 1 de la "cause de soi", cause interne par laquelle Spinoza définira la liberté (à la définition 7) et dans son déploiement intégral, notre béatitude (dans la partie V de l'Éthique), c'est dès la seconde définition d'Éthique I que Spinoza indique la limite externe qui, caractérisant tout individu fini, sera désignée, à partir d'Éthique III, comme son horizon de contrainte, de servitude et finalement de mort.

On peut ainsi affirmer que, si de la position d'un individu quelconque doit nécessairement suivre (de sa propre nature) des effets servant à sa conservation (selon la dynamique même du conatus qui n'enveloppe aucune négation, aucun temps fini mais une durée indéfinie...), il faut dire, aussi, que poser l'existence d'un individu quelconque c'est nécessairement poser, avec lui et en lui, une infinité d'autres individus qui font à la fois immédiatement obstacle à ce développement et, s'ils ne l'éliminent pas tout à fait, lui imposent, selon une nécessité contraignante, les chemins particuliers de son affirmation.

Cette affirmation s'étaye sur le scolie de la proposition 9 et la démonstration de la proposition 56, d'Éthique III. Premièrement, en effet: "l'appétit n'est rien d'autre que l'essence même de l'homme de la nature de laquelle suit nécessairement ce qui sert à sa conservation" (Éth. III, $9 \mathrm{sc}$ ), dans une continuation indéfinie de l'existence ; deuxièmement, "Quant au Désir il est l'essence même de chacun, ou sa nature, en tant qu'il est conçu comme déterminé à faire quelque chose par sa constitution, telle qu'elle est donnée (sc. de la prop 9); dès lors donc que chacun est affecté par des causes extérieures de telle ou telle espèce de Joie, de Tristesse, d'Amour, de Haine, c'est-à-dire dès lors que sa nature est constituée de telle façon ou de telle autre, son Désir sera nécessairement tel ou tel, et la nature d'un Désir différera de celle d'un autre Désir autant que les affections d'où ils naissent diffèrent entre elles" (Éth. III,, 56 dém). Ce qui signifie que les logiques de conserva- 
tion, qui se déduisent nécessairement de la nature déterminée de chaque individu, c'est-à-dire de son "penchant" ou de sa voluptas singulière ${ }^{30}$ sont plus ou moins adaptées, plus ou moins adéquates, voire plus ou moins délirantes...

Si pour Spinoza l'essence actuelle de l'individu est bien puissance d'affirmation et de conservation, cette puissance ne peut et ne doit donc se comprendre, dans la logique (et/ou la durée) de ses contenus déterminés, que selon l'articulation complexe et paradoxale - à la fois de vie et de mort (Éthique II, postulat 5 suivant la prop. 13; Éth. IV axiome) que tout individu entretient avec le monde extérieur. C'est dire aussi combien, au sein de rapports de forces nécessairement défavorables, pour tout mode fini voué à plus ou moins longue échéance à la destruction, l'affirmation absolue de sa propre causalité - comme causalité adéquate du conatus ou comme "libre nécessité" 31 - ou de "son propre droit" (TP II, 9; "droit absolu", dit Spinoza, qui permet d'être sui juris), cette affirmation est un combat.

Dans l'univers spinoziste nous ne sommes, dès notre naissance, que de manière infiniment partielle la cause de ce qui arrive en nous et de ce que nous faisons (Éth. III déf. 2). La seconde définition d'Éthique I enveloppe donc déjà un principe de contrariété qui sera explicitement posé dans la proposition 3 d'Éthique IV et par l'axiome mathématique sanglant qui ouvre cette partie IV de l'Éthique : 'Il n'est donné dans la Nature aucune chose singulière qu'il n'en soit donné une autre plus puissante et plus forte. Mais, si une chose quelconque est donnée, une autre plus puissante, par laquelle la première peut être détruite, est donnée".

C'est la position d'une condition de tout individu - y compris, bien sûr, le corps politique comme individu collectif-comme une condition de guerre totale dont tous seront nécessairement et successivement les victimes. Cette nécessité des rapports de forces (et par là même des jeux d'alliances, dans la résistance: ce n'est pas la guerre de tous contre tous!), cet horizon de guerre totale, auquel nul individu comme nulle société ne saurait se dérober, est proprement machiavélien.

\section{2) Nécessité, prudence, vertu: une ontologie du kairos}

La notion de nécessité a, chez Spinoza comme chez Machiavel, plusieurs significations, mais les deux penseurs se rencontrent dans le constat de la nécessité de cet état physique de fait de la guerre universelle. C'est la necessità à laquelle est confrontée l'action politique.

Contrairement en effet à Hobbes qui, par le contrat, croit annuler une fois pour toutes, l'état de nature, Spinoza écrit dans la lettre 50 à Jarig Jelles (datée du 2 juin 1674):

Vous me demandez quelle différence il y a entre Hobbes et moi quant à la politique: cette différence consiste en ce que je maintiens toujours le droit de nature et que je n'accorde dans une cité quelconque de droit au souverain sur les sujets que dans la mesure où, par la puissance, il l'emporte sur eux; c'est la continuation de l'état de nature. 
C'est-à-dire la continuation (par d'autres moyens... et pas toujours par d'autres moyens!) de l'état de guerre ou du droit de guerre, au sein même de la vie sociale et politique (TP III, 3). De ce point de vue, Spinoza rencontre aussi Machiavel sur la nécessité de la prudence (au sens du è necessario, "il est nécessaire" d'être prudent... du chapitre XV du Prince); ${ }^{32}$ nécessité de la prudence qui est appelée - pour Machiavel - par la nécessité (la necessità) des rapports de forces : "Il est nécessaire [è necessario] au Prince qui veut se conserver, qu'il apprenne à pouvoir n'être pas bon, et d'en user - ou n'user pas - selon la nécessité [secondo la necessità]". 33

Impératif de prudence, auquel sont tenus, le prince comme le peuple, s'ils veulent, chacun pour soi en tant qu' individus, conserver leur pouvoir ou leur liberté. Mais cet impératif de prudence n'est plus, pour Spinoza, de l'ordre du simple conseil, aussi utile et efficace soit-il, qui commanderait l'action sous la présupposition de conditions contingentes. ${ }^{34}$ Soit un impératif hypothétique ou technique engageant - à leur gré - les gouvernants raisonnables ou habiles à se donner, comme moyens de leur conservation, de bonnes stratégies... L'impératif de prudence est devenu en réalité, pour Spinoza, l'exigence vitale et immanente (aux rapports de forces) des conatus eux-mêmes : l'impératif ontologique de la durée.

La proposition 8 d'Éthique III affirme en effet: "L'effort par lequel chaque chose s'efforce de persévérer dans son être, n'enveloppe aucun temps fini, mais un temps indéfini”. Et sa démonstration:

Si en effet il enveloppait un temps limité qui déterminât la durée de la chose, il suivrait de la puissance même par où la chose existe, cette puissance étant considérée seule, qu'après ce temps limité la chose ne pourrait plus exister mais devrait être détruite; or cela (prop. 4) est absurde; donc l'effort par lequel la chose existe, n'enveloppe aucun temps défini; mais, au contraire, puisque (prop. 4), si elle n'est détruite par aucune cause extérieure, elle continuera d'exister par la même puissance par où elle existe actuellement, cet effort enveloppe un temps indéfini. ${ }^{3}$

Or cette "continuation indéfinie de l'existence" c'est précisément ce que, dans la définition 5 d'Éthique II, Spinoza appelle "la durée".

La nécessité logique qui nous fait passer de l'intelligence de la nécessité physique des rapports de forces, à la nécessaire prudence dans l'action politique, retrouve ainsi, sur un plan ontologique d'immanence, la nécessité même des conatus, leur dynamique affirmative et causale de conservation ou de duration, immanente aux rapports de forces. Non seulement, à la suite (et à l'exemple) de Machiavel, Spinoza dégage la prudence politique de toute fiction normative, et par là même de toute "obéissance" à une loi naturelle morale, mais il montre comment cette prudence, à laquelle chacun est nécessairement tenu par son droit naturel, procède de la nature même du processus d'individuation c'est-à-dire de l'essence actuelle de chaque individu. 
Ce qui signifie que la prudence est élevée, par Spinoza, à la vérité même de la logique de l'existant singulier, soit à la vérité de la logique même d'individuation, lorsque cette logique est comprise comme celle de la causalité adéquate de l'être fini.

Dans le Traité Politique, cette identification, entre la nécessité mathématique du droit de nature et l'impératif vital de prudence, est réalisée dans les articles 4, 5 et 6 du chapitre IV.

Rien n'est donc plus interdit au souverain, sauf ce qu'il ne peut pas décider sans encourir la destruction de sa propre "forme", c'est-à-dire sans soulever, contre lui, une indignation générale (TP III, 9) ou, suivant l'expression de Machiavel, sans s'attirer une "haine universelle". ${ }^{36}$ Le souverain étant, vis-à-vis des autres États (TP III, 11-13) et de son propre peuple, comme un individu est vis-à-vis d'un autre individu à l'état de nature, soit dans un strict rapport de puissance, il n'y a donc de sa part d'erreur que stratégique. ${ }^{37} \mathrm{C}$ 'est la seule manière par laquelle il puisse pécher, écrit Spinoza en TP II, 18: une mauvaise intelligence des rapports de forces le conduisant à une action inajustée, imprudente, dont les effets se retournent contre sa propre conservation. Il n'y a donc, en ce sens, de péché qu'envers soi-même, dans et par la logique d'une causalité inadéquate.

Chez Spinoza, c'est ainsi le réel lui-même de (et dans) la durée qui le constitue qui, adéquatement ou inadéquatement, selon la logique même des conatus individuels, est devenu machiavéliennement prudent. C'est-à-dire pratiques multiples stratégiques, contradictoires et/ou combinées, d'affirmation et de résistance dans et par les processus dynamiques d'individuations. Chaque individu, autant qu'il est en lui de puissance (quantum in se est), déduisant nécessairement de sa propre nature (et/ou de ses propres dispositions actuelles) et dans des conditions déterminées, cette logique de guerre (d'affirmation et de conservation) selon laquelle Machiavel souhaitait que le Prince conduise lucidement, intelligemment, son action politique. Et bien que le terme même de stratégie (de strâtegos, chef d'armée) n'existât pas encore dans son lexique, c'est bien, avec Machiavel, qu'est née véritablement une pensée stratégique comme, à la fois, théorie de la guerre naturelle et nécessaire afin d'assurer la conservation de l'État, mais aussi art politique de la prise du pouvoir et de sa conservation.

Et cette idée de la stratégie du réel qui vient, chez Spinoza comme chez Machiavel, nourrir d'une tout autre signification l'ancienne notion (aristotélicienne et thomiste) de prudence, est l'expression d'une prise de partie pour la rationalité et l'intelligibilité intégrale du réel dans ses différentes individuations; rationalité et intelligibilité aussi de l'action et de l'histoire, en dehors de toute croyance en leur téléologie naturelle. Ainsi, si la double thèse de la guerre perpétuelle et universelle qu'est le réel et la stratégie politico-militaire qu'elle nécessite est celle par laquelle Machiavel a le plus radicalement rompu avec la tradition politique de la prudence, cette thèse est aussi - et face aux mêmes adversaires - celle par laquelle Spinoza est le plus profondément machiavélien. 
Mais ne l'est-il pas plus radicalement encore que Machiavel lui-même, par cette extension interne de la nécessité stratégique de l'individuation à tout le réel en son essence, comme puissance déterminée actuelle et actualisante? On peut se poser une telle question en décelant toutefois que, chez Machiavel, la notion de virtù indique déjà cette direction philosophique.

Certes la nécessité apparaît tout d'abord chez Machiavel sous la forme de la contrainte, celle de la force des choses extérieures ou des aléas de la fortune sous le surplomb de laquelle les hommes doivent se décider promptement. ${ }^{38}$ Mais la virtù répond aussi à la contrainte non pas comme une ré-action mais comme une affirmation immanente aux rapports de forces: c'est une véritable activité de résistance. D'une part en effet, "la fortune démontre sa puissance aux endroits où il n'y a point de force dressée pour lui résister...", et cette force Machiavel la nomme virtù; ${ }^{39}$ d'autre part, "la vertu est la plus forte là où le choix a le moins joué". ${ }^{40}$ La virtù ne résiste donc pas réactivement à la nécessité, mais elle répond activement, adéquatement, stratégiquement à son exigence ${ }^{41}$ Obéir à la nécessité c'est, non pas agir de manière hétéronome - sous la contrainte des forces extérieures - mais c'est agir adéquatement suivant la logique d'un essentiel retrouvé; comme si la contrainte avait permis de purger l'action, de la purifier de ses illusions pour ramener, en quelque sorte, l'individu à son principe actif, son "principe vital", ${ }^{42}$ son essence: la vie elle-même comme virtù, puissance actuelle et actualisante d'affirmation et de conservation.

Cette expérience de la virtù résistante, puissante, sachant ruser avec la fortune, dans l'attente-active des occasions qui permettraient de la dompter, Machiavel l'a faite auprès de César Borgia. C'est cette pratique, en direct, d'une puissance en actes qui n'est rien d'autre que la stratégie de son affirmation selon une appropriation réelle du temps historique soumis à la durée concrète de la volonté d'un homme, que Machiavel s'efforce de théoriser dans Le Prince.

Théorie du conatus (et/ou de la volonté lorsque cet effort se rapporte à l'âme seule, Éth. III, 9 sc.) dit Spinoza qui, en Éthique IV définition 8, écrit:

Par vertu et puissance j'entends la même chose; (prop. 7 p. III) la vertu [virtus], en tant qu'elle se rapporte à l'homme, est l'essence même ou la nature de l'homme en tant qu'il a le pouvoir de faire certaines choses se pouvant connaître par les seules lois de sa nature.

Donc d'être sui juris; donc de déduire de sa propre nature "nécessairement ce qui sert à sa conservation" (Éth. III, 9 sc.); ou encore d'être cause adéquate (Éth. III, déf. 2). Obéir adéquatement, stratégiquement à la nécessité-contrainte c'est donc, en dernière analyse, obéir à soi-même, à sa propre vertu ou virtù affirmée dans et par un contexte déterminé de rapports de forces (la necessità machiavélienne).

Si la virtù est l'affirmation même de la puissance d'agir propre d'un individu, cela signifie qu'à la différence de Hobbes - pour qui la conservation individuelle est la finalité de toute action et la puissance seulement le moyen d'assurer cette conservation - pour Machiavel, comme pour Spinoza, puissance et conser- 
vation ne font qu'un dans et par l'affirmation même de la vertu. C'est la nature stratégique de la potentia-virtù découverte par Machiavel, reprise et développée par Spinoza dans une théorie du Droit de nature (et du Droit en général) comme affirmation même de cette puissance.

La virtù n'est plus alors à considérer seulement comme un art prudentiel de "choisir" des moyens adaptés à une fin (elle est la plus faible, dit Machiavel, là où le choix a le plus joué...): la prise du pouvoir et sa conservation par exemple - art de l'astutia, du stratagème, de la ruse et de l'habileté - cela c'est la leçon que tireront de Machiavel les politiques "machiavéliennes"! (Cf. MATHERON, 1986c). Mais la virtù est plutôt la nécessité même de la puissance (et/ou de l'affirmation/ résistante immanente à la nécessité des rapports de forces), puissance de déterminer des problèmes, d'en décider avec le plus haut degré de lucidité, de pertinence et de rationalité pratique, puissance donc d'occuper effectivement, au sein même de la nécessité du réel des rapports de forces, le lieu de la décision, c'est-à-dire d'élever la puissance propre d'un individu à la vérité ontologique du problème de son affirmation, soit à l'actualité de sa libre nécessité, à cette position de maîtrise qui est celle d'une stratégie parfaite. Cela c'est la leçon que va tirer Spinoza de sa lecture de Machiavel et qu'il va développer du point de vue de sa propre ontologie de la puissance. Ontologie qui devient ainsi une ontologie dynamique de la décision du problème, ou encore, dans l'actualisation absolue de la puissance en chaque occasion singulière (in tempore), une ontologie du kairos. ${ }^{43}$

Car le projet politique comme le projet éthique spinoziste, c'est effectivement d'élever les hommes comme les peuples à des stratégies rationnelles parfaites, en toutes situations, en toutes occasions. Stratégies entendues comme affirmation absolue de l'existence ou du droit, c'est-à-dire de la puissance ou de la vertu en acte. C'est cette conception du droit comme puissance actuelle et stratégique, immanente aux rapports de forces, qui permet à Spinoza d'intégrer explicitement, dans son champ philosophique propre, les analyses de Machiavel, mais qui le conduit aussi à ne s'engager qu'avec une certaine circonspection sur la signification dernière de l'œuvre du Grand Florentin.

\section{3) La leçon politique de Machiavel}

Revenons sur la forme conditionnelle sous laquelle Spinoza présente la leçon politique de Machiavel en Traité Politique V, 7:

De quels moyens un prince mû par le seul désir de domination doit user pour consolider et conserver son État, le très pénétrant Machiavel l'a montré en détail. Quant à la fin que poursuit cet auteur, elle n'apparaît pas clairement. S'il s'en est proposé une bonne, comme il faut le croire en parlant d'un homme sage, elle semble avoir été de montrer de quelle imprudence beaucoup font preuve en s'efforçant de détrôner un tyran alors que pourtant les causes qui font du prince un tyran, loin de pouvoir être supprimées sont d'autant plus fortes que le prince a plus de sujets de crainte. 
Ce qui se produit lorsque la multitude a déjà donné des preuves d'hostilité envers un prince et glorifie comme un haut fait ce qui est un parricide. En outre, il a peut-être voulu montrer combien une libre multitude doit se garder de confier totalement son salut à un seul homme. Car cet homme, à moins d'être empli de vanité et de se croire capable de plaire à tous, est forcé de redouter chaque jour quelque trahison. Par suite il lui faut se consacrer à sa sécurité et tendre luimême des pièges à la multitude, au lieu de veiller sur elle. Je penche plutôt vers cette interprétation de l'œuvre de cet homme si sage, puisque il est reconnu qu'il fut un partisan de la liberté pour la défense de laquelle il a également donné les conseils les plus salutaires.

Remarquons tout d'abord que cette leçon de Machiavel, Spinoza la reprendra intégralement tant en ce qui concerne le refus tactique du tyrannicide que le constat de la folle imprudence du peuple lorsqu'il désire installer un homme seul au pouvoir. ${ }^{44}$ Car un prince lucide, c'est-à-dire qui peut échapper aux illusions de la vanité pour ne se consacrer exclusivement qu'à la réalisation de son désir de domination, un tel prince "doit" (est tenu de...) se conduire exactement comme Machiavel le lui conseille. C'est la prudence même qui l'exige, c'est-à-dire la logique stratégique immanente de son propre conatus en tant que désir exclusif de domination. ${ }^{45}$

Machiavel a donc raison sur tous les points. Pourquoi alors cette prudence dans la formulation de l'article 7 du chapitre V? Spinoza s'interroge sans doute, et sans réponse claire à ses yeux, sur la puissance politique effective que Machiavel accorde au Prince. Certes le prince de Machiavel exerce, pour Spinoza, son pouvoir "à bon droit" et "au mieux", si l'on se place du double point de vue de sa situation de prince nouveau et de son "seul désir de domination". Mais, pour Spinoza, le prince ne peut pas exercer son pouvoir "au mieux" si l'on se place du point de vue de l'affirmation absolue de son propre droit et aussi du droit absolu du Corps politique. Car le prince est, de ce point de vue, réellement incapable de réaliser effectivement, en lui et par lui, la puissance de la multitude par laquelle se définit le droit absolu de l'État et, dans l'adéquation, le droit du souverain.

Si Machiavel croit que le prince, dans sa situation et selon son désir de domination, peut identifier le pouvoir absolu qu'il détient à un droit absolu, il se trompe. Si Machiavel a seulement voulu faire la théorie de la pratique rationnelle du prince prudent (principe prudente) ${ }^{46}$ dans les limites de sa situation et de son seul désir de domination, il a entièrement vu juste. Mais s'il a cru que cette stratégie de circonstance et cette raison instrumentale, pouvait s'identifier à la stratégie rationnelle du Corps politique lui-même, c'est-à-dire à son droit absolu, il a commis une grave erreur $\left(T P\right.$ VI, 5). ${ }^{47}$

Et cette erreur d'appréciation serait alors le symptôme d'une méconnaissance de la nature même du Droit de l'État dans son identité constitutive avec la puissance de la multitude. Que Machiavel ait accepté comme une mesure de 
sagesse et de prudence l'idée d'une solution dictatoriale lorsqu'il s'agit de ramener l'État à son principe, viendrait plutôt confirmer, aux yeux de Spinoza, cette méconnaissance... ${ }^{48}$ Comme le dit le Traité Politique X, 1:

qui s'efforce d'éviter les maux qui menacent l'État doit lui offrir des remèdes qui s'accordent avec sa nature et puissent se déduire de ses fondements; sans quoi l'on tombe de Charybde en Scylla. Certes il est vrai que tous, gouvernants comme gouvernés, doivent être retenus par la crainte du châtiment ou du supplice; on empêche ainsi le péché de jouir de l'impunité ou du profit. En revanche, il est certain aussi que si cette crainte est commune aux bons et aux méchants, l'État se trouve nécessairement en grand danger. Comme le pouvoir dictatorial est absolu, il ne peut pas ne pas être redoutable à tous [...] Certes, puisque le pouvoir du dictateur est absolument celui d'un roi, il n'est pas sans grand danger pour la république que l'État puisse de temps à autre se changer en monarchie - même si c'est pour une période aussi brève que l'on voudra. ${ }^{49}$

L'éloge s'accompagnerait donc d'une remarque critique d'importance. Spinoza doit pourtant savoir que Machiavel a tenté de surmonter la contradiction structurelle entre le pouvoir absolu et l'actualisation effective du droit politique d'un prince qui ne peut, lui, s'affirmer absolument, qu'à la condition que le prince exprime dans et par ses décisions, la puissance politique effective de la multitude. Machiavel a souligné, en effet, la nécessité historique et vitale du lien du prince et du peuple contre les grands. Mais on peut supposer, qu'aux yeux de Spinoza, Machiavel n'aurait seulement fait que montrer ce que le prince, en tant qu'individu solitaire (du fait même de son pouvoir "absolu") pouvait faire, au mieux de ses intérêts, pour conserver son pouvoir et sa vie, en s'alliant le peuple contre les grands, sur la base d'une situation politique, inchangée dans sa nature, et qui reste pour tous la pire (en équilibre très instable entre tyrannie absolue et révolte), aussi bien pour le prince lui-même, que pour la multitude, que pour l'État. ${ }^{50}$

Et ce n'est alors, qu'une réforme profondément démocratique de la monarchie qui pourrait réaliser institutionnellement cette adéquation nécessaire du pouvoir de décision du prince et son droit absolu, dans et par l'adéquation de ce droit avec l'affirmation de la puissance de la multitude. ${ }^{51}$ Et c'est ce que démontre Spinoza, dans les chapitres VI et VII du Traité Politique, par une sorte de réécriture démocratique et populaire du Prince, à partir de sa propre ligne philosophique d'immanence qui, dans le domaine politique, devient ligne de masse.

En substituant ainsi la vertu de l'État d'une monarchie populaire bien instituée, à la virtuosité-virtì ${ }^{52}$ du prince (même s'il a su se rendre populaire...) $)^{53}$ Spinoza, loin de s'éloigner de Machiavel, en radicalise au contraire, aussi bien sur le plan ontologique qu'institutionnel, ce qui est, à ses yeux, son enseignement le plus authentique. Et cela en traitant de la question de l'art politique et de la conservation de cet individu qu'est l'État, comme de l'individu du prince, sur le terrain d'une 
ontologie de l'affirmation absolue de la puissance. C'est ainsi que Spinoza élève Machiavel à la vérité ontologique du problème de cette affirmation, problème que l'auteur du Prince et des Discours avait certes déjà su bien poser, dans l'identité de la puissance et de la vertu, sans pourtant encore adéquatement le résoudre.

Toute l'éthique et toute la politique de Spinoza sont la formulation d'une réponse qui est aussi un prolongement. Le réalisme ontologique de la durée réalise donc bien le souci machiavélien de la conservation. Mais c'est en inscrivant ce souci, propre à l'être fini, dans le procès ontologique d'une productivité indéfinie du réel. Productivité qui se dit en autant de stratégies qu'il faut nous efforcerc'est l'impératif de prudence - de connaître et par là même aussi de nous approprier, dans et par la plus grande productivité, éthique et politique, de la liberte. ${ }^{54}$

\section{Notes}

${ }^{1}$ SPINOZA. Ép. 56 à H. Boxel. trad. Appuhn. t. IV. p. 300 : ed. Gebbardt, t. IV. p. 261.

${ }^{2}$ La philosophie est une activité, une "énergie, disait Épicure:, qui procure par des discours et des raisonnements la vie bienheureuse" (SEXTUS EMPIRICUS. Adversus Mathematicos, XI, 169, cité par M. Conche [ÉPICURE, 1992, p. 40-41]).

${ }^{3}$ A. Comte-Sponville est, avec G. Deleuze. le penseur contemporain chez qui la relation positive entre spinozisme et épicurisme est la plus fortement soulignée.

${ }^{4}$ Les deux suppositions (absurdes) qui permettraient aux hommes, selon Spinoza, de se maintenir indéfiniment dans l'existence, sont les conditions mêmes, inter-mondaines. de la vie des dieux épicuriens, Eth. IV, 4 dém.

${ }^{5} \mathrm{Cf}$. Lettres à Hérodote, 35, 37, 45 et 82 ; Pythoclès 84, 85; Ménécée. 123, 135. Et Diogène Laerce (1965, t. II, p. 219), qui affirme qu'Épicure "exerçait ses élèves a bien tenir en leur mémoire ses propres écrits".

${ }^{6} \mathrm{Cf}$. à ce propos notre étude: (BOVE, 1996, p. 211-236).

${ }^{7}$ Suivant l'expression de CICERON, De finibus, I, 17, 57, citée par M. Conche (ÉPICURE, 1992, p. 78 , note 2 ).

8 "En vivant le jour bienheureux qui est en même temps le dernier de ma vie, je t'écris ceci. Les douleurs de vessie et d'entrailles que j'endure sont telles qu'elles ne peuvent être plus grandes; mais elles sont contre-battues par la joie de l'âme au souvenir de nos raisonnements et de nos entretiens passes" - Lettre à Idoménée citée par M. Conche (ÉPICURE, 1992, p. 92). Comme l'écrit GUYAU (1886, p. 197), "l'épicurien en se renfermant ainsi en lui-même, en cherchant ce qu'il y a de meilleur dans sa vie passée, y trouvera une force de résistance”.

De la Nature IV, 877-906, p. 140-141.

${ }^{10} \mathrm{Il}$ serait cependant schématique d'opposer une philosophie spinoziste du progrès à une philosophie épicurienne essentiellement marquée par le conservatisme d'un état primitif naturel. J. M. Guyau (1886) a même montré avec justesse que l'épicurisme a été l'un des rares courants philosophiques de l'Antiquité qui, contre l'esprit religieux, a cru l'homme susceptible de progrès; "l'épicurisme, [écrit-il], a affirmé (l') existence (du progrès) et, autant qu'on pouvait le faire à son époque, il l'a démontrée" (GUYAU, 1886, p. 166), Pourtant Épicure et Lucrèce sont victimes d'un état de fait. D'une part de l'absence en leur temps d'une réelle conscience de la transformation significative et irréversible du monde par la production humaine et historique. Pour Lucrèce, non la science en général mais seule la science épicurienne du bonheur possède une force de transformation humaine irréversible pour ceux qui l'utilisent. D'autre part de l'absence de la reconnaissance d'une valeur éthico-politique du progrès qui est au contraire perçu par les épicuriens sous son aspect pervers puisqu'il produit aussi, en dehors des choses réellement utiles, des désirs aussi illusoires qu'illimités. C'est ainsi que Lucrèce, après avoir montré ses bienfaits, en vient à blâmer les progrès de l'industrie et des arts et même à condamner "l'art funeste de la navigation"... Épicure et Lucrèce font alors d'une imitation historique une limite théorique de droit imposée par un concept de nature, pour eux, indépassable. Ainsi la science n'est-elle pas encore articulée à la dimension éthico-politique du monde et à cette seconde nature dont elle est la force productive.

${ }^{11}$ De la Nature V, 1430 p. 193 \& VI, 25 p. 199.

${ }_{12}$ "Il est, pour Spinoza, de la nature de la Raison, de percevoir les choses comme possédant une 
certaine sorte d'éternité" (Eth. II, 44, coroll 2), et non, comme pour Épicure, d'en percevoir les limites. La reconnaissance de ses propres limites est, chez Spinoza, soit une "impuissance" (Eth. III, 54 dem., III, 55 \& dem., IV, 53 dém.), soit une reconnaissance, par la connaissance adéquate, de ce qui, de l'extérieur, nous limite. C'est la connaissance d'un rapport de forces qui nous est défavorable, bien que cette connaissance vienne par ailleurs seconder notre puissance de résistance et d'affirmation.

${ }^{13}$ De la Nature VI, 18, p. 199.

${ }_{14}$ "Notre entendement serait plus imparfait si l'Âme était seule et qu'elle ne connût rien en dehors d'elle-même" (Eth. IV, 18 sc.).

${ }^{15}$ Nous avons consacré une étude, intitulée "Hilaritas et Acquiescentia in se ipso", Actes du Colloque de Jérusalem, d'avril 1993, sur la partie IV de l'Éthique; Spinoza by 2000, The Jerusalem Conferences, Spinoza on Reason and the 'Free Man' (Ethica IV), éd. E. J. Brill 2007; on trouve une traduction portugaise de cette étude : - "Hilaridade e contentamento intimo", in Psicopatologia: Clinicas de Hoje, trad. portuguaise et éd. de David Calderoni (org.), éd. Via lettera, Sào Paulo (Brésil) 2006. Ce texte constitue le chapitre IV de La Stratégie du conatus (BOVE, 2012[1996]).

${ }^{16}$ Lettre à Ménécée, 127, M. Conche (ÉPICURE, 1992, p. 221).

${ }^{17}$ On serait ici tenté d'écrire que pour Épicure, comme pour Spinoza, absolument parlant, la sagesse est "méditation non de la mort mais de la vie" (Eth, IV, 67). Mais l'expression n'est pas tout à fait valable chez Épicure pour qui la mort est nécessairement incluse dans les pactes de l'homme avec la nature (les foedera naturae). Ce qui n'est pas le cas chez Spinoza pour qui l'alliance avec la Nature (qui est de connaissance et d'amour de Dieu) ne peut être qu'affirmation "éternelle... de la vie" à l'exclusion de toute méditation sur la mort. Du point de vue de Spinoza, la durée propre du sage épicurien (faite de gratitude envers le passé et de confiance en l'avenir) semblerait ainsi en rester au plan d'une continuité psychologique sans atteindre à la continuité ontologique de la durée de l'éternité que pourtant elle vise.

${ }^{18}$ Sentences Vaticanes, 10, attribuée à Métrodore (trad. Solovine), non retenue par M. Conche.

${ }^{19}$ Lettre à Ménécée, 143, M. Conche (ÉPICURE, 1992, p. 227).

${ }^{20}$ Sentences Vaticanes, 41, M. Conche (ÉPICURE, 1992, p. 257).

${ }^{21}$ Lettre à Ménécée, 142, M. Conche (ÉPICURE, 1992, p. 227).

${ }^{22}$ Dans le Traité Politique, Machiavel, dont les thèses sont présentes tout au long de l'œuvre, est explicitement cité deux fois: chapitre $\mathrm{V}$ article 7 et chapitre $\mathrm{X}$ article 1.

${ }^{23}$ Sur la notion de prudence chez Spinoza, cf. notre ouvrage en portugais (BOVE, 2010, p. 6376). Cf. aussi notre conférence donnée à la PUC le 28 août 2012 : "La prudence des corps chez Spinoza. De la physique à l'histoire" (à paraître dans la revue Conatus).

${ }^{24} \mathrm{Cf}$. la fin de la lettre 57 à Hugo Boxel.

${ }^{25}$ Le Prince, chapitre IX, "De la principauté civile" p. 317 de l'éd. de la Pléiade. Cf. aussi Discours sur la première décade de Tite-Live, livre I ch. IV p. 390.

${ }^{26}$ Deux "humeurs" (dua umori diversi) ou encore deux "appétits différents" (dua appetiti diversi), Le Prince ch. IX p. 317 (pour le texte italien nous nous servons de l'éd. de la Biblioteca Universale Rizzoli in Classici della Bur, Franco Melotti et Ettore Janni, introduction de R. Aron, 1994).

${ }^{27}$ Discours livre I ch. I p. 381. Pertanto, non potendo gli uomini assicurarsi se non con la potenza, è necessario fuggire questa sterilità del paese, e porsi in luoghi fertilissimi, dove potendo per la libertà del sito ampliare, possa e difendersi da chi l'assaltasse e opprimere qualunque alla grandezza sua si opponesse (éd. Biblioteca Universale Rizzoli, Giorgio Inglese, intr. de Gennaro Sasso, p. 62, 1996). Cf. aussi Discours, livre III ch. XLV p. 713: la règle générale de toute existence c'est, avant tout, de "soutenir son premier choc".

${ }^{28}$ On ne remarque pas assez que la notion de conatus est tout d'abord utilisée, par les auteurs latins, dans un contexte essentiellement guerrier ; cf. par exemple César in De la guerre civile, conatus adversariorum infringere $(2,21,1)$, ou Tite-Live dans son Histoire de Rome, cum frusta multi conatus ad erumpendum capti essent $(9,4,1)$; in ipso conatu gerendi belli $(32,28,4)$; in ipso conatu rerum $(9,18,14) \ldots$

${ }^{29}$ Discours, livre I ch. XXXVII p. 461.

${ }^{30}$ Son propre principe de plaisir en quelque sorte; trahit sua quemque voluptas, TP II, 6 (la formule est reprise de Virgile, Bucoliques, II,65. Elle est proche de Lucrèce, De natura rerum, II, 172). Pour Machiavel, cf. Discours, livre III ch. IX p. 641-642.

${ }^{31}$ Que l'effort puisse devenir causalité adéquate, cela signifie que l'essence de son action, comme effet, peut s'expliquer ou totalement se définir par l'essence de l'être quelconque (qui fait effort)

Fractal, Rev. Psicol., v. 24 - n. 3, p. 443-472, Set./Dez. 2012 
comme cause, comme permet de l'affirmer l'axiome 2 de la partie V de l'Éthique. Pour un mode fini, ce devenir causalité adéquate ne pouvant s'effectuer toujours, nécessairement, que selon certaines relations. Pour l'expression "libre nécessité" cf. lettre 58 à G.H. Schuller, Appuhn IV p. 304.

${ }^{2}$ Onde è necessario a un principe, volendosi mantenere, imparare a potere essere non buono, $e$ usarlo e non usare secondo la necessità, Il Principe ch. XV, 1994, p. 147.

${ }^{33}$ Le Prince, ch. XV p. 335, éd. Pléiade.

${ }^{34}$ Suivant les "avertissements" que donne Machiavel et que le prince prudent devrait entendre, $\mathrm{Le}$ Prince, ch. XXIV, p. 363, éd. Pléiade.

${ }^{35}$ Nous citons l'Éthique dans la traduction Appuhn en 4 volumes G.F.

${ }^{36}$ Discours, livre III ch. VI p. 618. Pour Spinoza l'indignation est "une Haine envers quelqu'un qui a fait du mal à un autre" (Éthique III déf. 20 des Affects).

${ }^{37}$ Cf. déjà Machiavel, Le Prince, ch. XII p. 325.

${ }^{38}$ Cf. Discours, livre III, ch. VI p. 636.

${ }^{39}$ Le Prince ch. XXV p. 365.

${ }^{40}$ Discours livre I ch. I p. 380.

${ }_{41}$ "Je répète donc, comme une vérité incontestable et dont les preuves sont partout dans l'histoire, que les hommes peuvent seconder la fortune et non s'y opposer; ourdir les fils de sa trame et non les briser. Je ne crois pas pour cela qu'ils doivent s'abandonner eux-mêmes. Ils ignorent quel est son but; et comme elle n'agit que par des voies obscures et détournées, il leur reste toujours l'espérance; et dans cette espérance, ils doivent puiser la force de ne jamais s'abandonner, en quelque infortune et misère qu'ils puissent se trouver", Discours livre II ch. XXIX p. 597.

${ }^{42}$ Dans Discours, livre III ch. I, Machiavel parle des institutions et des hommes qui, par leur vertu, ont été capables de ramener l'ensemble des autres hommes "au principe vital de la république... verso il suo principio", Pléiade p. 609, éd. Bibl. Universale Rizzoli p. 462.

${ }^{43} \mathrm{La}$ prudence élevée à sa vérité de principe ontologique de la durée, c'est l'exercice même de la fortitudo (la Force d'Ame) qui, dans la Fermeté (Animositas) fait que l'homme "s'efforce à se conserver en vertu du seul commandement de la Raison" en faisant preuve de la plus haute "Présence d'Esprit dans les périls... animi in periculis prcesentia" (Éth. III, 59 sc.); "Dans un homme libre donc la fuite opportune [in tempore] et le combat témoignent d'une égale Fermeté d'âme ; autrement dit, l'homme libre choisit la fuite avec la même Fermeté d'âme, ou présence d'esprit [animi prcesentia], que le combat" (Éth. IV, 69 coroll.). Dans Le Prince, le rapport talent/ occasion est déjà celui de l'affirmation de la puissance singulière (comme virtù), capable de s'élever à la vérité du problème, dans une situation particulière ; cf. dans le ch. VI p. 304, les exemples de Moïse, Romulus, Cyrus, Thésée...

${ }^{44}$ Cf. Discours, livre I ch. XL, l'exemple d'Appius p. 472 sq.

45 "Un monarque d'institution récente tentera donc de toutes ses forces d'établir des lois nouvelles, de réformer à son profit les droits existant dans l'Etat, et de réduire le peuple à une condition telle qu'il ne puisse reprendre aux rois leur dignité aussi facilement que la leur Donner", écrit Spinoza dans le chapitre XVIII du Traité Théologico-politique (Appuhn II p. 308, Geb. III p. 226). Spinoza - à la différence de Machiavel - ne pose pas explicitement le problème du projet plus lointain du prince dominateur qui n'a pas, en lui-même, le désir "personnel" de domination mais bien "l'intérêt commun et celui de la patrie plutôt que le sien propre et celui de ses héritiers"; cf. Discours, livre I ch. IX et les exemples de Romulus, Lycurgue, Solon... et de Moïse. Il est vrai que, de fait, Moïse est, chez Spinoza aussi, un tel homme.

${ }^{46}$ Le Prince, ch. XXIII, Pléiade p. 361; éd. Bibl. Universale Rizzoli p. 182.

${ }^{47} \mathrm{Si}$ l'on en croit cependant le livre I des Discours ch. LVIII, Machiavel est bien conscient du contraire (cf. particulièrement la p. 505).

${ }^{48}$ Cf. Discours, livre I ch. XVIII p. 429-431, et le ch. XXXIV.

${ }^{49}$ Cet article, ainsi que ceux suivant le chapitre X, discute les options machiavéliennes des Discours.

${ }^{50}$ Il est vrai que Machiavel enseigne que "le meilleur moyen" qu'un prince "puisse employer est de se concilier l'amitié du peuple" (Discours, livre I ch. XVI p. 424-426). Pour cela le prince doit répondre, d'une certaine manière, aux désirs de ce peuple, $1^{\circ}$ ) en réprimant les grands (satisfaisant ainsi la vengeance d'un peuple qui pense que les grands sont causes de son esclavage); $2^{\circ}$ ) en répondant au désir de liberté du petit nombre, soit en élevant certains citoyens aux places de commandement... ou en les éliminant. Le plus grand nombre ne désirant, au fond, être libre "que pour vivre en sécurité, c'est exclusivement à ce second désir que le prince doit s'efforcer de répondre en éradiquant ainsi, de fait, le désir de liberté. Car le prince, pour Machiavel, ne 
peut pas réellement satisfaire le désir du peuple de conserver sa liberté. Le pouvoir du prince et la liberté sont incompatibles. C'est ce que confirme Spinoza lorsqu'il écrit dans le ch. XVIII du TTP, que "le roi en maintenant les droits antiques du peuple paraîtrait plutôt son esclave que son maître" (Appuhn II p. 308, Geb. III p. 226). Le Traité Politique montrera que seule une monarchie réformée, dans le sens de la démocratie, peut lever cette contradiction entre le peuple et le prince, la liberté et la sécurité. Que le peuple seul puisse imposer "as" monarchie au prince, c'est ce que montrait déjà aussi le chapitre XXXV du livre I des Discours (mais pour un temps limité, car le peuple est facilement corrompu par les manœuvres du tyran). Sur l'alliance intéressée du prince avec le peuple, cf. l'exemple d'Appius dans le ch. XL du livre I des Discours (p. 472 sq.). De toutes manières, du fait des périls encourus, le "Prince nouveau" devra être nécessairement très cruel (ou du moins paraître tel), Le Prince, ch. XVII p. 338 sq.

${ }^{51}$ Dans Le Prince, Machiavel écrit que "Les principaux fondements qu'aient tous les États, aussi bien les nouveaux que les anciens et les mixtes, sont les bonnes lois et bonnes armes"; mais, poursuit-il, "je laisserai de parler des lois et traiterai des armes" (ch. XII p. 324). Spinoza reprend la question des lois (que Machiavel traite aussi, en partie, dans les Discours: Romulus se fait attribuer une autorité exclusive pour "donner de bonnes lois", Discours I, ch. IX p. 405-406).

${ }^{2}$ Le Prince, ch. VI p. 304.

${ }^{3}$ Le Prince, ch. IX: "il est nécessaire [è necessario] qu'un prince se fasse aimer de son peuple: autrement il n'a remède aucun en ses adversités". A la fin du chapitre Machiavel parlera d'un "prince sage" [principe savio], Pléiade p. 319 ; éd. Bibl. Universale Rizzoli p. 124.

${ }^{54}$ Dans le domaine politique, ce travail de connaissance des stratégies du réel, Spinoza a commencé à l'effectuer dans le Traité Théologico-politique par l'étude de l'État hébreu. Et c'est l'Écriture, dans son apport d'expérience historique, qui lui offre, d'abord, un modèle de stratégie parfaite ou - suivant l'expression de Machiavel dans le chapitre I de la partie III des Discours -, un modèle de "prudence intrinsèque" du corps commun. Or, ce modèle est paradoxal en ce qu'il nous éclaire sur l'auto-organisation du corps collectif mais à partir de l'étude de conditions qui inscrivent d'abord ce corps, en régime de radicale hétéronomie. Car avec l'étude de l'État hébreu c'est la dimension corporelle de l'imagination qui est pointée au cœur de la politique et de l'histoire et, avec elle, le problème de son rapport constituant aux habitudes communes et aux confiances partagées. Car l'ontologie de la durée enveloppe nécessairement une dynamique de l'habitude et de ses contractions comme elle enveloppe aussi, nécessairement, une confiance qui est son essence même (cf. à propos de l'analyse de l'État Hébreu le chapitre 9 de notre Espinoza e a psicologia social (BOVE, 2010, p. 125-134).

\section{RÉFÉRENCES}

BOVE, L. Épicurisme et spinozisme: 1'éthique. Archives de Philosophie, Paris, t. 57, cahier 3, p. 471-484, juil.-sept. 1994.

BOVE, L. Enseignement du Christ et résistance dans le Traité ThéologicoPolitique de Spinoza. In: GROS, G. (Org.). La Bible et ses raisons. Saint-Étienne, France: Université de Saint-Étienne, 1996. p. 211-236. Ouvrage collectif de l'UPRES-A 5037.

BOVE, L. (Éd.) Une ontologie politique de la durée: Spinoza lecteur de Machiavel. In: Introduction: De la prudence des corps. Du physique au politique. In: Spinoza, Traité Politique. Paris: Librairie générale française, 2002. p. 31-46. Collection Le Livre de Poche.

BOVE, L. Potêntia e prudêntia de uma vida como singularidade em Espinoza. In: . Espinoza e a psicologia social, Ensaios de ontologia politica e antropogênese. Belo Horizonte: Autêntica, 2010. p. 63-76. 
BOVE, L. Dall'innata potestas alle strategie del conatus, o l'epicureismo in moto perpetuo. In: DEL LUCCHESE, F.; MORFINO, V.; MORMINO, G. (Cur.). Lucrezio e la modernità. I secoli XV-XVII. Napoli: Bibliopolis, 2011. p. 163-178.

BOVE, L. La Stratégie du conatus: affirmation et résistance chez Spinoza (1996). Paris: Vrin, 2012.

BROCHARD, V. La théorie du plaisir d'après Épicure. In: _. Études de philosophie ancienne et de philosophie moderne. Paris: Vrin, 1912. p. 252-293.

CESAR, J. La guerre civile. Texte établi et trad. par Pierre Fabre. Paris: Les Belles Lettres, 1997.

COMTE-SPONVILLE, A. Le mythe d'Icare: traité du désespoir et de la Béatitude. Paris: PUF, 1984.

DELEUZE, G. Lucrèce et le simulacre. In: . Logique du Sens. Paris: Minuit, 1969.

ÉPICURE. Lettres et Maximes. Trad. par Marcel Conche. Paris: PUF, 1992.

GUYAU, J. M. La morale d'Épicure et ses rapports avec les doctrines contemporaines. Paris: Alcan, 1886.

LAERCE, D. Vie, Doctrines et Sentences des Philosophes Illustres. Paris: Garnier-Flammarion, 1965. t. II, X, 31, p. 264.

LUCRÈCE, C. De la Nature II, 60. Trad. par H. Clouard. Paris: GarnierFlammarion, 1964.

MACHIAVEL, N. De la principauté civile. In: Le Prince. In: BARINCOU, E. (Ed.). Machiavel: Oeuvres complètes. Paris: Gallimard, 1952a. Collection: Bibliothèque de la Pléiade.

MACHIAVEL, N. Discours sur la première décade de Tite-Live. In: BARINCOU, E. (Ed.). Machiavel: Oeuvres complètes. Paris: Gallimard, 1952b. Collection: Bibliothèque de la Pléiade. Livre I, ch. IV.

MACHIAVEL, N. Il Principe. Milano: Biblioteca Universale Rizzoli, 1994. Series Classici della Bur.

MACHIAVEL, N. Discorsi sopra la prima deca di Tito Livio. Milano: Biblioteca Universale Rizzoli, 1996. Series Classici della Bur.

MATHERON, A. Spinoza et la sexualité. In: . Anthropologie et Politique 
au XVIIe siècle: études sur Spinoza. Paris: Vrin, 1986a. p. 209-230. Texte republié dans Alexandre Matheron, Études sur Spinoza et les philosophies classiques, ENS Éditions p. 305-324.

MATHERON, A. L'anthropologie spinoziste? In: . Anthropologie et Politique au XVIIe siècle: études sur Spinoza. Paris: Vrin, 1986b. p. 175-185. (étude republiée dans Études sur Spinoza, p. 15-24).

MATHERON, A. Spinoza et la décomposition de la Politique thomiste: Machiavélisme et Utopie. In: . Anthropologie et Politique au XVIIe siècle: études sur Spinoza. Paris: Vrin, 1986c. p. 49-79. (étude republiée dans Études sur Spinoza, p. 81-111).

SPINOZA, B. Ép. 56 à H. Boxel (1674). Oeuvres IV: Traité politique, Lettres. Trad. Charles Appuhn. Paris: GF Flammarion, 1966.

SPINOZA, B. Oeuvres III: Éthique. Trad. Charles Appuhn. Paris: GF Flammarion, 1993.

SPINOZA, B. Traité politique (1677). Trad. Emile Saisset revisée par Laurent Bove. Paris: Le Livre de Poche, 2002.

SPINOZA, B. Oeuvres III: Traité théologico-politique (1670). Trad. Jacqueline Lagrée et Pierre-François Moreau. Paris: PUF, 2009.

TITE-LIVE. Histoire Romaine. Texte établi et trad. Paul Jal. Paris: Les Belles Lettres, 2005.

VIRGILE. Bucoliques. Texte établi et trad. par E. de Saint-Denis. Paris: Les Belles Lettres, 1949.

Reçu: Octobre 10, 2012 Accepté: Novembre 17, 2012 
